\title{
Lista das espécies de Culicidae (Diptera) depositadas na Coleção de Entomologia Pe. J. S. Moure ${ }^{1}$
}

\author{
Ana Cristina Tissot ${ }^{2,3} \&$ Mario Antonio Navarro da Silva ${ }^{2,3}$
}

${ }^{1}$ Contribuição n ${ }^{\circ} 1708$ do Departamento de Zoologia da Universidade Federal do Paraná.

${ }^{2}$ Departamento de Zoologia, Pós-graduação da Entomologia, Universidade Federal do Paraná (UFPR), Centro Politécnico, Caixa Postal 19020, 81531-980 Curitiba-PR. anatissot@ hotmail.com; mnavarro@ufpr.br

${ }^{3}$ Bolsista CNPq.

\begin{abstract}
Species list of the Culicidae (Diptera) deposited at the Entomological Collection "Pe. J. S. Moure". The Entomological Collection of Departamento de Zoologia of Universidade Federal do Paraná (DZUP) accommodates about 5,000,000 insect specimens. The collection of Diptera is represented by approximately 2,000,000 specimens, being Muscidae, Culicidae and Syrphidae the most representative families. The Diptera order consists of approximately 150,000 described species and in the Collection about 103,000 specimens within 78 families are registered. The list of species of the family Culicidae presents 9,579 specimens within 2 subfamilies, 7 tribes, 17 genera, 26 subgenera and 85 species. Most specimens were captured in forest remnants in urban and rural areas, wild and urban environments in coastal regions or urban areas of different cities of Paraná State, including some species captured in the States of São Paulo, Mato Grosso and Santa Catarina and at Distrito Federal. The specific information of each specimen, such as collection site, latitude, longitude, collector, collection date, collection method and the specialist's name who identified it, as well as taxonomic data (order, tribe, genus, subgenus and species), was included in a electronic data base.
\end{abstract}

KEYWORDS. Brazil; mosquitoes; taxonomy.

\begin{abstract}
RESUMO. Lista das espécies de Culicidae (Diptera) depositadas na Coleção de Entomologia Pe. J. S. Moure. A Coleção de Entomologia do Departamento de Zoologia da Universidade Federal do Paraná (DZUP) abriga cerca de 5.000.000 de exemplares de insetos. A coleção de Diptera está representada por aproximadamente 2.000 .000 de exemplares, sendo Muscidae; Culicidae e Syrphidae as famílias mais representativas. A ordem Diptera possui cerca de 150.000 espécies descritas e a coleção conta com cerca de 103.000 exemplares representantes de 78 famílias. A lista das espécies pertencentes à família Culicidae conta com 9.579 exemplares, distribuídos em 2 subfamílias, 7 tribos, 17 gêneros, 26 subgêneros e 85 espécies. A maioria dos exemplares foi coletado em remanescentes de mata localizados em áreas urbanas e rurais, ambientes silvestres e urbanos de regiões litorâneas ou áreas urbanas pertencentes a diferentes cidades do Estado do Paraná, e algumas espécies incluídas foram capturadas nos Estados de São Paulo, Mato Grosso, Santa Catarina e no Distrito Federal. As informações específicas de cada exemplar, como local de coleta, latitude, longitude, coletor, data de coleta, método de coleta e nome do pesquisador que identificou, e também informações taxonômicas como ordem, tribo, gênero, subgênero e espécie, foram informatizados em um banco de dados.
\end{abstract}

PALAVRAS-CHAVE. Brasil; mosquitos; taxonomia.

A Coleção de Entomologia Pe. J. S. Moure (DZUP), iniciada em 1956, do Departamento de Zoologia da Universidade Federal do Paraná abriga cerca de 5 milhões de exemplares de insetos e cerca de 3.000 exemplares-tipo. A coleção de Diptera está representada por aproximadamente 2 milhões de exemplares fixados em álcool ou a seco em mantas, alfinetados ou em lâminas, cerca de $40 \%$ identificados ao nível genérico ou específico. As famílias mais representativas são Muscidae, Culicidae e Syrphidae. Apesar da coleção encontrar-se melhor representada pela dipterofauna do Paraná, possui razoável número de exemplares da Bahia, Minas Gerais, Mato Grosso, São Paulo, Santa Catarina e Rio Grande do Sul, bem como de outros países em algumas poucas famílias.

Até a década de 70 os dípteros eram pouco representados na coleção, especialmente pela falta de pesquisadores especialistas no grupo. Atualmente a coleção conta com cerca de 103.000 exemplares representantes de 78 famílias (Marinoni et al. 2005). Em recente cooperação com a Faculdade de Saúde Pública da Universidade de São Paulo os culicídeos resultantes das pesquisas daquela instituição estão sendo depositados no DZUP, promovendo assim o crescimento substancial da coleção de referência na área de Entomologia Médica.

$\mathrm{Na}$ coleção de culicídeos estão preservados, a seco, espécimes adultos fêmeas e machos, montados em alfinete entomológico ou em lâminas com a genitália, e espécimes imaturos, exúvias de larvas e pupas montados em lâminas permanentes e com identificação semelhante ao adulto correspondente. Para a catalogação das espécies um banco de dados foi criado, no programa Acces, contendo informações específicas de cada exemplar, como local de coleta, latitude, longitude, coletor, data de coleta, método de coleta e nome do pesquisador que identificou, além de informações taxonômicas como ordem, tribo, gênero, subgênero e espécie.

$\mathrm{O}$ acervo conta com 9.579 exemplares, distribuídos em duas 
subfamílias, sete tribos, 17 gêneros, 26 subgêneros e 85 espécies, a maioria coletados em remanescentes de mata localizados em áreas urbanas e rurais, ambientes silvestres e urbanos de regiões litorâneas ou áreas urbanas pertencentes a diferentes cidades do Paraná e algumas espécies coletadas em outros estados como São Paulo, Mato Grosso, Santa Catarina e no Distrito Federal.

A elaboração da lista da coleção com amostras da culicidofauna paranaense permite a catalogação das espécies que ocorrem no estado e conseqüentemente a delimitação de áreas de transmissão de arbovírus; protozoários e filárias, e também a comparação de amostras que ocorrem no estado com as de outras áreas de ocorrência de determinado grupo, tornando possível a análise da variabilidade morfológica das espécies, o reconhecimento de novas espécies ou de complexos de espécies.

Na lista das espécies de culicídeos depositadas na coleção os espécimens foram apresentados em ordem alfabética de subfamílias, seguidas das respectivas tribos, gêneros e espécies, localidade em que foram coletadas e número total de exemplares adultos fêmeas (f) e machos (m); existindo lâminas de imaturos estas foram citadas também com o número total de fêmeas (f) e machos (m).

\section{ANOPHELINAE \\ ANOPHELINI}

GÊNERO Anopheles Meigen, 1818

Anopheles (Anopheles) eiseni Coquillett, 1902

Brasil, PARANÁ, Morretes, Instituto Ambiental do Paraná II (IAPAR) $3 \mathrm{f}$.

Anopheles (Anopheles) fluminensis Root, 1927

Brasil, PARANÁ, Morretes, Instituto Ambiental do Paraná II (IAPAR) $4 \mathrm{f}$

Anopheles (Anopheles) intermedius (Peryassú, 1908)

Brasil, PARANÁ, Morretes, Instituto Ambiental do Paraná II (IAPAR) $1 \mathrm{f}$.

Anopheles (Anopheles) mediopunctatus (Lutz, 1903)

Brasil, PARANÁ, Morretes, Instituto Ambiental do Paraná II (IAPAR) $3 \mathrm{f}$.

Anopheles (Anopheles) peryassui Dyar \& Knab, 1908

Brasil, DISTRITO FEDERAL, Brasília, Bananal, Convenio Florestal $1 \mathrm{f}$.

Anopheles (Kerteszia) cruzii Dyar \& Knab, 1908

Brasil, PARANÁ, Piraquara, Mananciais da Serra, carvalho $4 \mathrm{f}$, Mata $2 \mathrm{f}$.

Brasil, PARANÁ, Morretes, Instituto Ambiental do Paraná II (IAPAR) $64 \mathrm{f}$.

Anopheles (Nyssorhynchus) albitarsis Lynch Arribálzaga, 1878

Brasil, PARANÁ, Curitiba, Centro Integrado de Atendimento ao Adolescente Infrator (CIAADI) $5 \mathrm{f}$.

Anopheles (Nyssorhynchus) evansae (Brèthes, 1926)

Brasil, PARANÁ, Morretes, Instituto Ambiental do Paraná II (IAPAR) $3 \mathrm{f}, 1 \mathrm{~m}$.

Anopheles (Nyssorhynchus) lutzii Cruz, 1901

Brasil, PARANÁ, Morretes, Instituto Ambiental do Paraná II (IAPAR) $2 \mathrm{f}$.

Anopheles (Nyssorhynchus) parvus (Chagas, 1907)

Brasil, PARANÁ, Morretes, Instituto Ambiental do Paraná II (IAPAR) $1 \mathrm{f}$.

Brasil, DISTRITO FEDERAL, Brasília, Bananal, Convenio Florestal 2 f. Anopheles (Nyssorhynchus) strodei Root, 1926

Brasil, DISTRITO FEDERAL, Brasília, Bananal, Convenio Florestal $2 \mathrm{f}$.

Brasil, PARANÁ, Curitiba, Museu de História Natural do Capão da Imbuia (MHNCI) $1 \mathrm{f}$.

\section{CULICINAE}

\section{AEDEOMYIINI}

GÊNERO Aedeomyia Theobald, 1901

Aedeomyia (Aedeomyia) squamipennis (Lynch Arribálzaga, 1878)

Brasil, PARANÁ, Curitiba, Parque Regional do Iguaçu $3 \mathrm{f}$.

Brasil, PARANÁ, Morretes, Instituto Ambiental do Paraná II (IAPAR) $3 \mathrm{f}$.

\section{AEDINI}

\section{GÊNERO Aedes Meigen, 1818}

Aedes (Ochlerotatus) crinifer (Theobald, 1903)

Brasil, PARANÁ, Curitiba, Centro Integrado de Atendimento ao Adolescente Infrator (CIAADI) 16 f e 2 m, Museu de História Natural do Capão da Imbuia (MHNCI) 41 f, Jardim Botânico 10f. Brasil, PARANÁ, Piraquara, Mananciais da Serra, mata peridomicílio $1 \mathrm{~m}$, trilha Cayugava $17 \mathrm{f}$ e $39 \mathrm{~m}$, carvalho $2 \mathrm{~m}$.

Aedes (Ochlerotatus) fluviatilis (Lutz, 1904)

Brasil, PARANÁ, Curitiba, Centro Integrado de Atendimento ao Adolescente Infrator (CIAADI) $8 \mathrm{f}$ e $1 \mathrm{~m}$, Vila Fanny $7 \mathrm{f}$ e $8 \mathrm{~m}$, Boqueirão $3 \mathrm{f} \mathrm{e} 3 \mathrm{~m}$, Alto Boqueirão $6 \mathrm{f} \mathrm{e} 3 \mathrm{~m}$, Guabirotuba $5 \mathrm{f} \mathrm{e}$ $1 \mathrm{~m}$, Sítio Cercado $6 \mathrm{f} \mathrm{e} 1 \mathrm{~m}$, BR $11612 \mathrm{f} \mathrm{e} 1 \mathrm{~m}$, BR 116 Xaxim 9 f e 2 m, Jardim Botânico 17 f, Museu de História Natural do Capão da Imbuia (MHNCI) $1 \mathrm{f}$.

Aedes (Ochlerotatus) fulvus (Wiedemann, 1828)

Brasil, PARANÁ, Ilha do Mel $1 \mathrm{f}$.

Aedes (Ochlerotatus) hastatus Dyar, 1922

Brasil, PARANÁ, Piraquara, Mananciais da Serra, trilha Cayugava $1 \mathrm{~m}$.

Aedes (Ochlerotatus) nubilus Theobald, 1903

Brasil, PARANÁ, Piraquara, Mananciais da Serra, trilha Cayugava $14 \mathrm{~m}$.

Aedes (Ochlerotatus) oligopistus Dyar, 1918

Brasil, PARANÁ, Piraquara, Mananciais da Serra, trilha Cayugava $1 \mathrm{f} \mathrm{e} 2 \mathrm{~m}$.

Aedes (Ochlerotatus) rhyacophilus Costa Lima, 1933

Brasil, PARANÁ, Morretes, Instituto Ambiental do Paraná II (IAPAR) $21 \mathrm{f}$.

Aedes (Ochlerotatus) scapularis (Rondani, 1848)

Brasil, PARANÁ, Curitiba, Parque Regional do Iguaçu 20 f, Centro Integrado de Atendimento ao Adolescente Infrator (CIAADI) 75 f e $1 \mathrm{~m}$, Museu de História Natural do Capão da Imbuia (MHNCI) $258 \mathrm{f}$ e $6 \mathrm{~m}$

Brasil, PARANÁ, Piraquara, Mananciais da Serra, trilha Cayugava $32 \mathrm{f}$ e $8 \mathrm{~m}$, carvalho $2 \mathrm{f}$ e $2 \mathrm{~m}$, mata interior $1 \mathrm{f}$, mata $1 \mathrm{f}$.

Brasil, PARANÁ, Morretes, Instituto Ambiental do Paraná II (IAPAR) $18 \mathrm{f}$.

Brasil, PARANÁ, Dr. Camargo, Recanto Marista 4 f.

Aedes (Ochlerotatus) serratus (Theobald, 1901)

Brasil, PARANÁ, Curitiba, Parque Regional do Iguaçu 4 f, Jardim Botânico 1 f, Museu de História Natural do Capão da Imbuia (MHNCI) $15 \mathrm{f}$.

Brasil, PARANÁ, Piraquara, Mananciais da Serra, trilha Cayugava $9 \mathrm{f}$, carvalho $2 \mathrm{f}$.

Brasil, PARANÁ, Morretes, Instituto Ambiental do Paraná II (IAPAR) $14 \mathrm{f}$.

Brasil, PARANÁ, Dr. Camargo, Recanto Marista $8 \mathrm{f}$.

Aedes (Protomacleaya) terrens (Walker, 1856)

Brasil, PARANÁ, Piraquara, Mananciais da Serra, carvalho $3 \mathrm{f}$, mata interior $1 \mathrm{f}$.

Brasil, PARANÁ, São José dos Pinhais, área rural 24 f e 16 m.

Brasil, PARANÁ, Curitiba, Museu de História Natural do Capão da Imbuia (MHNCI) $1 \mathrm{f}$.

Aedes (Stegomyia) aegypti (Linnaenus, 1762)

Brasil, PARANÁ, Curitiba,Uberaba $23 \mathrm{f}$ e $11 \mathrm{~m}$.

Brasil, MATO GROSSO, Coxipó 2 f e $6 \mathrm{~m}$.

Aedes (Stegomyia) albopictus (Skuse, 1894)

Brasil, PARANÁ, Curitiba, Parque Regional do Iguaçu $1 \mathrm{f}$ e $1 \mathrm{~m}$, Museu de História Natural do Capão da Imbuia (MHNCI) 1 f, Santa Cândida 8 f e 7 m. 
Brasil, SÃO PAULO, Registro, área urbana 76 f e $47 \mathrm{~m}$. Brasil, SANTA CATARINA, São Francisco do Sul 4 f.

GÊNERO Haemagogus Williston, 1896

Haemagogus (Haemagogus) capricornii Lutz, 1904 Brasil, SÃO PAULO, Porto Cabral, Rio Paraná $1 \mathrm{f}$.

Haemagogus (Conopostegus) leucocelaenus (Dyar \& Shannon, 1924) Brasil, PARANÁ, Curitiba, Museu de História Natural do Capão da Imbuia (MHNCI) 55 f e 25 m/lâminas 11 f e 4 m, Jardim Botânico $14 \mathrm{f}$.

GÊNERO Psorophora Robineau-Desvoidy, 1827

Psorophora (Grabhamia) confinnis (Lynch Arribálzaga, 1891)

Brasil, PARANÁ, Morretes, Instituto Ambiental do Paraná II (IAPAR), mata $6 \mathrm{f}$, aberto $50 \mathrm{f}$

Brasil, PARANÁ, Morretes, Instituto Ambiental do Paraná II (IAPAR) $1 \mathrm{f}$

Psorophora (Grabhamia) cingulata (Fabricius, 1805)

Brasil, PARANÁ, Morretes, Instituto Ambiental do Paraná II (IAPAR), mata $370 \mathrm{f} \mathrm{e} 14 \mathrm{~m}$, aberto $78 \mathrm{f}$.

Brasil, PARANÁ, Morretes, Instituto Ambiental do Paraná II (IAPAR) $31 \mathrm{f}$.

Psorophora (Janthinosoma) albipes (Theobald, 1907)

Brasil, PARANÁ, Dr. Camargo, Recanto Marista $12 \mathrm{f}$.

Psorophora (Janthinosoma) ferox (Von Humboldt, 1819)

Brasil, PARANÁ, Curitiba, Parque Regional do Iguaçu 542 f, Jardim Botânico 3 f, Museu de História Natural do Capão da Imbuia (MHNCI) 18 f/lâminas 12 f.

Brasil, PARANÁ, Piraquara, Mananciais da Serra, trilha Cayugava $40 \mathrm{f}$, carvalho $1 \mathrm{f}$.

Psorophora (Janthinosoma) lutzii (Theobald, 1901)

Brasil, PARANÁ, Morretes, Instituto Ambiental do Paraná II (IAPAR), mata $9 \mathrm{f}$, aberto $2 \mathrm{f}$ e $1 \mathrm{~m}$.

Psorophora (Psorophora) ciliata (Fabricius, 1794)

Brasil, PARANÁ, Curitiba, Centro Integrado de Atendimento ao Adolescente Infrator (CIAADI) $1 \mathrm{f}$.

Brasil, SÃO PAULO, Ribeirão Preto, Fazenda da Pedra $1 \mathrm{f}$.

\section{CULICINI}

GÊNERO Culex Linnaeus, 1758

Culex (Culex) acharistus Root, 1927

Brasil, PARANÁ, Curitiba, Parque Regional do Iguaçu 148 f, Museu de História Natural do Capão da Imbuia (MHNCI) 3 f/lâminas 3 f.

Brasil, PARANÁ, Piraquara, Mananciais da Serra, trilha Cayugava $1 \mathrm{f}$, mata $1 \mathrm{~m}$.

Culex (Culex) bidens Dyar, 1922

Brasil, PARANÁ, Curitiba, Parque Regional do Iguaçu 35 f, Centro Integrado de Atendimento ao Adolescente Infrator (CIAADI) 10 f e $4 \mathrm{~m}$, Museu de História Natural do Capão da Imbuia (MHNCI) $26 \mathrm{f} \mathrm{e} 1 \mathrm{~m}$.

Brasil, PARANÁ, Piraquara, Mananciais da Serra, trilha Cayugava $1 \mathrm{~m}$, peridomicílio $1 \mathrm{f}$.

Culex (Culex) chidesteri Dyar, 1921

Brasil, PARANÁ, Curitiba, Centro Integrado de Atendimento ao Adolescente Infrator (CIAADI) 5 f, Museu de História Natural do Capão da Imbuia (MHNCI) $7 \mathrm{f}$.

Culex (Culex) coronator Dyar \& Knab, 1906

Brasil, PARANÁ, Curitiba, Parque Regional do Iguaçu 86 f, Centro Integrado de Atendimento ao Adolescente Infrator (CIAADI) 3 f, Museu de História Natural do Capão da Imbuia (MHNCI) 97 f e 3 m/lâminas 4 f e 2 m, BR116 Xaxim 5 f e 4 m, Jardim Botânico $240 \mathrm{f} \mathrm{e} 1 \mathrm{~m}$.

Brasil, PARANÁ, Piraquara, Mananciais da Serra, trilha Cayugava $3 \mathrm{f} \mathrm{e} 1 \mathrm{~m}$.

Brasil, PARANÁ, Morretes, Instituto Ambiental do Paraná II (IAPAR) $57 \mathrm{f}$.

Culex (Culex) declarator Dyar \& Knab, 1906

Brasil, PARANÁ, Curitiba, Jardim Botânico 1 f.
Brasil, PARANÁ, Piraquara, Mananciais da Serra, trilha Cayugava $3 \mathrm{f} \mathrm{e} 1 \mathrm{~m}$.

Culex (Culex) dolosus (Lynch Arribálzaga, 1891)

Brasil, PARANÁ, Piraquara, Mananciais da Serra, trilha Cayugava $3 \mathrm{~m}$.

Culex (Culex) eduardoi Casal \& Garcia, 1968

Brasil, PARANÁ, Curitiba, Jardim das Américas 49 f e 61 m, Centro Politécnico $12 \mathrm{f}$ e $4 \mathrm{~m}$.

Brasil, PARANÁ, Morretes $5 \mathrm{f}$ e $1 \mathrm{~m} /$ lâminas $4 \mathrm{f}$.

Culex (Culex) lygrus Root, 1927

Brasil, PARANÁ, Curitiba, Centro Integrado de Atendimento ao Adolescente Infrator (CIAADI) $1 \mathrm{~m}$, Museu de História Natural do Capão da Imbuia (MHNCI) $2 \mathrm{f}$.

Brasil, PARANÁ, Piraquara, Mananciais da Serra, trilha Cayugava $2 \mathrm{~m}$.

Culex (Culex) nigripalpus Theobald, 1901

Brasil, PARANÁ, Curitiba, Centro Integrado de Atendimento ao Adolescente Infrator (CIAADI) 3 f, Museu de História Natural do Capão da Imbuia (MHNCI) $7 \mathrm{f}$.

Brasil, PARANÁ, Piraquara, Mananciais da Serra, trilha Cayugava $4 \mathrm{f}$.

Brasil, PARANÁ, Morretes, Instituto Ambiental do Paraná II (IAPAR) $3 \mathrm{f}$

Culex (Culex) quinquefasciatus Say, 1823

Brasil, PARANÁ, Curitiba, Centro Integrado de Atendimento ao Adolescente Infrator (CIAADI) 169 f e 13 m, Museu de História Natural do Capão da Imbuia (MHNCI) 610 f e 508 m / lâminas 41 f e $24 \mathrm{~m}$, BR116 Xaxim $10 \mathrm{f}$ e $7 \mathrm{~m}$, BR116 Bairro Alto $2 \mathrm{f}$ e $3 \mathrm{~m}$, Jardim das Américas $3 \mathrm{f}$ e $2 \mathrm{~m}$, Centro Politécnico $1 \mathrm{f}$.

Culex (Culex) usquatus Dyar, 1918

Brasil, PARANÁ, Piraquara, Mananciais da Serra, trilha Cayugava $1 \mathrm{f} \mathrm{e} 2 \mathrm{~m}$.

Culex (Melanoconion) atratus Theobald, 1901

Brasil, PARANÁ, Morretes, Instituto Ambiental do Paraná II (IAPAR) $14 \mathrm{f}$.

Culex (Melanoconion) misionensis Duret, 1953

Brasil, PARANÁ, Morretes, Instituto Ambiental do Paraná II (IAPAR) $54 \mathrm{f}$.

Culex (Melanoconion) pedroi Sirivanakarn \& Belkin, 1980

Brasil, PARANÁ, Morretes, Instituto Ambiental do Paraná II (IAPAR) $2 \mathrm{f}$.

Culex (Melanoconion) pilosus (Dyar \& Knab, 1906)

Brasil, PARANÁ, Piraquara, Mananciais da Serra, trilha Cayugava $2 \mathrm{f} \mathrm{e} 2 \mathrm{~m}$

Culex (Melanoconion) ribeirensis Forattini \& Sallum, 1985

Brasil, PARANÁ, Morretes, Instituto Ambiental do Paraná II (IAPAR) $56 \mathrm{f}$.

Culex (Melanoconion) zeteki Dyar, 1918

Brasil, PARANÁ, Morretes, Instituto Ambiental do Paraná II (IAPAR) $14 \mathrm{f}$.

Culex (Microculex) elongatus Rozeboom \& Komp, 1950

Brasil, PARANÁ, Piraquara, Mananciais da Serra, carvalho 2 f e 9 $\mathrm{m}$, mata interior $1 \mathrm{f}$.

Culex (Microculex) imitator Theobald, 1903

Brasil, PARANÁ, Piraquara, Mananciais da Serra, trilha Cayugava $5 \mathrm{f} \mathrm{e} 4 \mathrm{~m}$, carvalho $3 \mathrm{f}$ e $8 \mathrm{~m}$, mata interior $4 \mathrm{f}$ e $5 \mathrm{~m}$, mata $4 \mathrm{f} \mathrm{e}$ $5 \mathrm{~m}$, mata peridomicílio $1 \mathrm{~m}$.

Culex (Microculex) microphyllus Root, 1927

Brasil, PARANÁ, Piraquara, Mananciais da Serra, carvalho $4 \mathrm{f}$ e 5 $\mathrm{m}$, trilha Cayugava $1 \mathrm{f}$, mata interior $6 \mathrm{f}$ e $2 \mathrm{~m}$, mata $1 \mathrm{f}$.

Culex (Microculex) neglectus Lutz, 1904

Brasil, PARANÁ, Piraquara, Mananciais da Serra, mata interior $1 \mathrm{f}$ e $2 \mathrm{~m}$, mata peridomicílio $1 \mathrm{~m}$, carvalho $7 \mathrm{~m}$, mata $2 \mathrm{~m}$.

Culex (Microculex) pleuristriatus Theobald, 1903

Brasil, PARANÁ, Curitiba, Santa Cândida 4 f e 5 m, Museu de História Natural do Capão da Imbuia (MHNCI) $1 \mathrm{~m} /$ lâmina $1 \mathrm{~m}$.

Culex ocellatus Theobald, 1903

Brasil, PARANÁ, Piraquara, Mananciais da Serra, carvalho $2 \mathrm{~m}$, mata $3 \mathrm{f}$ e $3 \mathrm{~m}$. 
GÊNERO Lutzia Theobald, 1903

Lutzia (Lutzia) bigoti (Bellardi, 1862)

Brasil, PARANÁ, São José dos Pinhais, área rural 7 f e 9 m.

\section{MANSONIINI}

GÊNERO Coquillettidia Dyar, 1905

Coquillettidia (Rhynchotaenia) chrysonotum (Peryassu, 1922)

Brasil, PARANÁ, Morretes, Instituto Ambiental do Paraná II (IAPAR), mata $7 \mathrm{f}$, aberto $1 \mathrm{f}$.

Brasil, PARANÁ, Piraquara, Mananciais da Serra, trilha Cayugava $3 \mathrm{f}$.

Coquillettidia (Rhynchotaenia) chrysonotum (Peryassu, 1922) / Coquillettidia (Rhynchotaenia) albifera (Prado, 1931)

Brasil, PARANÁ, Morretes, Instituto Ambiental do Paraná II (IAPAR) $6 \mathrm{f}$.

Coquillettidia (Rhynchotaenia) nigricans (Coquillett, 1904)

Brasil, PARANÁ, Morretes, Instituto Ambiental do Paraná II (IAPAR) $1 \mathrm{f}$.

Coquillettidia (Rhynchotaenia) venezuelensis (Theobald, 1912)

Brasil, PARANÁ, Curitiba, Parque Regional do Iguaçu 7 f, CIAADI

296 f e 4 m, Museu de História Natural do Capão da Imbuia (MHNCI) $48 \mathrm{f} \mathrm{e} 1 \mathrm{~m}$.

Brasil, PARANÁ, Morretes, Instituto Ambiental do Paraná II (IAPAR) $26 \mathrm{f} \mathrm{e} 2 \mathrm{~m}$, mata $325 \mathrm{f} \mathrm{e} 11 \mathrm{~m}$, aberto $42 \mathrm{f}$ e $1 \mathrm{~m}$.

Brasil, Paraná, Morretes 39 f e 13 m.

GÊNERO Mansonia Blanchard, 1901

Mansonia (Mansonia) fonsecai (Pinto, 1932)

Brasil, PARANÁ, Curitiba, Parque Regional do Iguaçu 3.379 f, Centro Integrado de Atendimento ao Adolescente Infrator (CIAADI) 1 f, Museu de História Natural do Capão da Imbuia (MHNCI) $4 \mathrm{f}$.

Mansonia (Mansonia) iguassuensis Barbosa, Navarro-Silva \& Sallum, 2007 - HOLÓTIPO

Brasil, PARANÁ, Curitiba, Parque Regional do Iguaçu 60 f, Centro Integrado de Atendimento ao Adolescente Infrator (CIAADI) 3f.

\section{SABETHINI}

GÊNERO Limatus Theobald, 1901

Limatus durhamii Theobald, 1901

Brasil, PARANÁ, Curitiba, Parque Regional do Iguaçu 1 f, Santa Cândida $5 \mathrm{f}$.

Brasil, PARANÁ, Piraquara, Mananciais da Serra, trilha Cayugava $2 \mathrm{f}$.

Brasil, PARANÁ, São José dos Pinhais, área rural 77 f.

Brasil, PARÁ, Piratuba $3 \mathrm{f}$.

Limatus flavisetosus Oliveira Castro, 1935

Brasil, PARANÁ, Curitiba, Museu de História Natural do Capão da Imbuia (MHNCI) $1 \mathrm{f}$ /lâmina $1 \mathrm{f}$.

GÊNERO Onirion Harbach \& Peyton, 2000

Onirion personatum (Lutz, 1904)

Brasil, PARANÁ, Piraquara, Mananciais da Serra, carvalho $10 \mathrm{f}$, mata $2 \mathrm{f}$.

Onirion personatum (Lutz, 1904) / Onirion brucei Lutz, 1904 (Del Ponte \& Cerqueira, 1938)

Brasil, PARANÁ, Piraquara, Mananciais da Serra, carvalho $6 \mathrm{f}$, mata $1 \mathrm{f}$.

GÊNERO Runchomyia Theobald, 1903

Runchomyia (Runchomyia) cerqueirai Stone, 1944

Brasil, PARANÁ, Curitiba, Museu de História Natural do Capão da Imbuia (MHNCI) $1 \mathrm{f}$.

Runchomyia (Runchomyia) reversa Lane \& Cerqueira, 1942 Brasil, PARANÁ, Piraquara, Mananciais da Serra, mata peridomicílio $2 \mathrm{f}$, carvalho $14 \mathrm{f}$, mata interior $1 \mathrm{f}$, mata $8 \mathrm{f}$.

Brasil, PARANÁ, Morretes, Instituto Ambiental do Paraná II (IAPAR) $2 \mathrm{f}$.

Runchomyia (Runchomyia) theobaldi Lane \& Cerqueira, 1942 Brasil, PARANÁ, Piraquara, Mananciais da Serra, carvalho $6 \mathrm{f}$.
GÊNERO Sabethes Robineau-Desvoidy, 1827

Sabethes (Peytonulus) aurescens (Lutz, 1905)

Brasil, PARANÁ, Piraquara, Mananciais da Serra, carvalho $14 \mathrm{f}$, mata interior $2 \mathrm{f}$, mata $2 \mathrm{f}$.

Brasil, PARANÁ, Curitiba, Museu de História Natural do Capão da Imbuia (MHNCI) $1 \mathrm{f}$.

Sabethes (Peytonulus) undosus (Coquillett, 1906)

Brasil, PARANÁ, Piraquara, Mananciais da Serra, mata interior $1 \mathrm{f}$. Sabethes (Sabethes) purpureus (Theobald, 1907)

Brasil, PARANÁ, Curitiba, Museu de História Natural do Capão da Imbuia (MHNCI) $2 \mathrm{f}$.

Sabethes (Sabethinus) xhyphydes Harbach, 1994

Brasil, PARANÁ, Piraquara, Mananciais da Serra, mata interior 1 f, carvalho $2 \mathrm{f}$.

Sabethes (Sabethinus) intermedius (Lutz, 1904)

Brasil, PARANÁ, Piraquara, Mananciais da Serra, carvalho $2 \mathrm{f}$, mata interior $1 \mathrm{f}$, mata $2 \mathrm{f}$.

GÊNERO Shannoniana Theobald, 1903

Shannoniana (Shannoniana) fluviatilis (Theobald, 1903)

Brasil, PARANÁ, Piraquara, Mananciais da Serra, carvalho $44 \mathrm{f}$, trilha Cayugava $1 \mathrm{f}$, mata $3 \mathrm{f}$.

Brasil, PARANÁ, Morretes, Instituto Ambiental do Paraná II (IAPAR) $1 \mathrm{f}$.

GÊNERO Trichoprosopon Theobald, 1901

Trichoprosopon compressum Lutz, 1905

Brasil, PARANÁ, Piraquara, Mananciais da Serra, carvalho $1 \mathrm{f}$.

Trichoprosopon pallidiventer (Lutz, 1905)

Brasil, PARANÁ, Curitiba, Parque Regional do Iguaçu 18 f, Jardim Botânico Jardim Botânico 2 f.

Brasil, PARANÁ, Piraquara, Mananciais da Serra, carvalho 74 f, mata $13 \mathrm{f}$ e $2 \mathrm{~m}$, trilha Cayugava $1 \mathrm{f}$.

Brasil, PARANÁ, Morretes, Instituto Ambiental do Paraná II (IAPAR) $1 \mathrm{f}$.

GÊNERO Wyeomyia Theobald, 1901

Wyeomyia (Phoniomyia) antunesi Lane \& Guimarães, 1937

Brasil, PARANÁ, Piraquara, Mananciais da Serra, borda de mata $2 \mathrm{f}$, mata interior $2 \mathrm{f}$, carvalho $8 \mathrm{f}$, mata $5 \mathrm{f}$.

Wyeomyia (Phoniomyia) edwardsi Lane \& Cerqueira, 1942

Brasil, PARANÁ, Piraquara, Mananciais da Serra, carvalho $9 \mathrm{f}$, mata interior $2 \mathrm{f}$, mata $1 \mathrm{f}$.

Wyeomyia (Phoniomyia) galvaoi (Correa \& Ramalho, 1956)

Brasil, PARANÁ, Piraquara, Mananciais da Serra, peridomicílio $1 \mathrm{f}$, mata interior $1 \mathrm{f}$, mata $1 \mathrm{f}$.

Wyeomyia (Phoniomyia) pallidoventer (Theobald, 1907)

Brasil, PARANÁ, Curitiba, Museu de História Natural do Capão da Imbuia (MHNCI) $2 \mathrm{f}$.

Wyeomyia (Phoniomyia) quasilongirostris (Theobald, 1907)

Brasil, PARANÁ, Curitiba, Parque Regional do Iguaçu 17 f, Jardim Botânico 2 f.

Brasil, PARANÁ, Piraquara, Mananciais da Serra, trilha Cayugava $1 \mathrm{f}$, carvalho $3 \mathrm{f}$, mata $1 \mathrm{f}$.

Wyeomyia (Wyeomyia) limai Lane \& Cerqueira, 1942

Brasil, PARANÁ, Piraquara, Mananciais da Serra, mata $4 \mathrm{f}$, carvalho $7 \mathrm{f}$.

Wyeomyia mystes Dyar, 1924 / Wyeomyia finlayi Lane \& Cerqueira, 1942 Brasil, PARANÁ, Piraquara, Mananciais da Serra, mata interior $1 \mathrm{f}$, carvalho $1 \mathrm{f}$.

\section{URANOTAENIINI}

GÊNERO Uranotaenia Lynch Arribálzaga, 1891

Uranotaenia (Uranotaenia) calosomata Dyar \& Knab, 1907 Brasil, PARANÁ, Piraquara, Mananciais da Serra, trilha Cayugava $2 \mathrm{f}$. Brasil, PARANÁ, Morretes, Instituto Ambiental do Paraná II (IAPAR) $3 \mathrm{f}$.

Uranotaenia (Uranotaenia) lowii Theobald, 1901

Brasil, PARANÁ, Piraquara, Mananciais da Serra, mata interior $1 \mathrm{f}$. 


\section{REFERENNCIAS}

Barbosa, A. A.; M. A. N. Silva \& M. A. M. Sallum. 2007. Mansonia (Mansonia) iguassuensis sp. nov. (Diptera: Culicidae) from Brazil. Zootaxa 1527: 45-52.

Bellardi, L. 1862. Saggio di ditterologia messicana. Apêndice 1 pl., Torino $28 \mathrm{p}$.

Blanchard, R. 1901. Observations sur quelques moustiques. Comptes Rendus des Séances et Mémoires de la Société de Biologie 53: $1045-1046$.

Brèthes, J. 1926. Description provisoire de deux especes nouvelles d'Anophelinae argentins. Prensa Medica Argentina 13: 106107.

Casal, O. H. \& M. Garcia. 1968. Culex (Culex) eduardoi uma nueva espécie de la Republica Argentina (Diptera, Culicidae). Revista de la Sociedad Entomológica Argentina 30: 105-111.

Chagas, C. 1907. Novas espécies de Culicidios Brazileiros Trabalho do Instituto de Manguinhos, Besnard Frères, Rio de Janeiro $28 \mathrm{p}$.

Coquillett, D. W. 1902. New forms of Culicidae from North America. Journal of the New York Entomological Society 10: 191194.

Coquillett, D. W. 1904. New North American Diptera. Proceedings Entomological Society of Washington 6: 166-192.

Coquillett, D. W. 1905 (1906). New Culicidae from the West Indies and Central America. Proceedings Entomological Society of Washington 7: 182-186.

Correa, R. R. \& G. R. Ramalho. 1956. Revisão de Phoniomyia Theobald, 1903 (Diptera, Culicidae, Sabethini). Folia Clinica et Biologica 25: $1-176$.

Costa Lima, A. M. da. 1933. Sobre um novo Aedes (Ochlerotatus) do Brasil. Memórias do Instituto Oswaldo Cruz 27: 403-406.

Cruz, O. G. 1901. Contribuição para o estudo dos culicídeos do Rio de Janeiro. Brazil-Medico 15: 423-426.

Del Ponte, E. \& N. Cerqueira. 1938. Alguns Sabethineos do Brasil (Diptera, Culicidae). Revista de Entomologia 8: 225-237.

Duret, J. P. 1953. Contribucion al conocimento de la distribuicion geografica de los culicidos argentinos (Diptera-Culicidae). Revista de La Sanidad Militar Argentina 30: 372-388.

Dyar, H. G. 1905. Remarks on genitalic genera in the Culicidae. Proceedings Entomological Society of Washington 7: 4249.

Dyar, H. G. 1918. The male genitalia of Aedes as indicative of natural affinities (Diptera, Culicidae). Insecutor Inscitiae Menstruus 6: $71-86$.

Dyar, H. G. 1918. New American Mosquitoes (Diptera, Culicidae). Insecutor Inscitiae Menstruus 6: 120-129.

Dyar, H. G. 1921. Ilustrations of certain mosquitoes (Diptera, Culicidae). Insecutor Inscitiae Menstruus 9: 114-118.

Dyar, H. G. 1922. The American Aedes of the serratus group (Diptera, Culicidae). Insecutor Inscitiae Menstruus 10: 157-166.

Dyar, H. G. 1922. Notes on tropical American mosquitoes (Diptera, Culicidae). Insecutor Inscitiae menstruus 10: 188-196.

Dyar, H. G. 1924. A new Sabethid from Brazil (Diptera, Culicidae). Insecutor Inscitle Menstruus 12: 91-92.

Dyar, H. G. \& F. Knab. 1906. The larvae of Culicidae classified as independent organisms. Journal of the New York Entomological Society 14: 169-243.

Dyar, H. G. \& F. Knab. 1907. Descriptions of new mosquitoes from the Panama Canal Zone. Journal New York Entomological Society 15: $197-210$.

Dyar, H. G. \& F. Knab. 1908. Descriptions of some new mosquitoes from Tropical America. Proceedings of the United States National Museum 35: 53-70.

Dyar, H. G. \& R. C. Shannon. 1924. The subfamilies, tribes, and genera of American Culicidae. Journal of the Washington Academy of Sciences 14: 472-486.

Fabricius, J. C. 1794. Entomologia systematica emendacta et aucta.
Vol. 4, Hafniae [=Copenhagen] $472 \mathrm{p}$

Fabricius, J. C. 1805. Systema antliatorum secundum ordines, genera, species, Brunsvigae [=Brunswick] $373 \mathrm{p}$.

Forattini, O. P. \& M. A. M. Sallum. 1985. A new species of Culex (Melanoconion) from Southern Brazil (Diptera:Culicidae). Revista de Saúde Pública 19: 171-182.

Guimarães, J. H. 1997. Systematic database of Diptera of the Americas South of the United States (Family Culicidae) Editora Plêiade, 286 p.

Harbach, R. E. 1994. The subgenus Sabethinus of Sabethes (Diptera:Culicidae). Systematic Entomology 19: 207-234.

Lane, J. \& N. L. Cerqueira. 1942. Os Sabetíneos da América (Diptera, Culicidae). Arquivos de Zoologia 3: 473-849.

Lane, J. \& L. R Guimarães. 1937. Notas sobre culicídeos de Campos do Jordão. Annaes Paulistas de Medicina e Cirurgia 22: 211317.

Linnaeus, C. 1758. Systema naturae per regna tria naturae. Editio $\mathrm{X}$, vol.1, Holmiae $824 \mathrm{p}$.

Linnaeus, C. 1762. Zweyter Theil, enthalt Beschreibungen verchiedener wuchtiger Naturalien. Pp. 267-606. In: Hasselquist, F., Reise nach Palestina in den Jahren von 1749 bis 1752 Rostock, Germany $606 \mathrm{p}$.

Lutz, A. 1904. Quadros dos gêneros da família Culicidae. 2 p. In: Bourroul. Mosquitos do Brasil, Tese de Doutorado. 78 p.

Lutz, A. 1905. Novas espécies de mosquitos do Brasil. Imprensa Medica 13: 26-29, 65-69, 81-83, 101-104, 125-128, 169-172, 202-203, 269-271, 287-290, 311-314, 347, 350.

Lynch Arribálzaga, F. 1878. Descripcion de três nuevos Culicidae de Buenos Aires. El Naturalista argentino: revista de historia natural 1: 149-153.

Lynch Arribálzaga, F. 1891. Dipterología argentina. Revista del Museo de La Plata 1: $345-377$.

Marinoni L.; M. S. Couri; L. M. Almeida; J. Grazia; G. A. R. Melo. 2005. Coleções entomológicas brasileiras: estado da arte e perspectivas para dez anos. Documento de trabalho. Projeto Diretrizes e Estratégias para a Modernização de Coleções Biológicas Brasileiras e a Consolidação de Sistemas Integrados de Informações sobre Biodiversidade, disponível em http://www.cria.org.br/cgee/ documentos/ColecoesEntomologicas.doc, acessado em Junho de 2007.

Meigen, J. W. 1818. Systematische Beschreibung der bekannten europäischen zweiflügeligen Insekten. Vol.1, Hamm. xxxvi +333 pp.

Oliveira Castro, G. M. de. 1935. Estudo sobre uma espécie de "Limatus" que se cria em buracos de pau (Dipt., Culicidae). Revista do Departamento Nacional de Produção Animal 2: 143-155.

Peryassú, A. G. 1908. Os culicidios do Brazil. Instituto de Manguinhos. Rio de Janeiro, Brasil, 407 p.

Peryassu, A. G. 1922. Uma nova espécie de culicineo brasileiro. A Folha Medica 3: $117-118$.

Pinto, C. 1932. Alguns mosquitos do Brasil e do oriente da Bolívia (Diptera, Culicidae). Memórias do Instituto Oswaldo Cruz 40: $285-309$.

Prado, A. 1931. Contribuições ao conhecimento dos culicídeos de São Paulo. II. Notas sobre as espécies encontradas nos arredores da capital e sobre a determinação de Aedes crinifer (Theob.) Trabalho da secção de Protozoologia e Parasitologia do Instituto Butantan, 6p.

Robineau-Desvoidy, J. B. 1827. Essai sur la tribu dês culicides. Memoirs du Museum d'Histoire Naturelle 3: 390-413.

Rondani, C. 1848. Esame di varie specie d'insetti ditteri brasiliani. Studi Entomologici 1: $63-112$.

Root, F. M. 1926. Studies on Brazilian mosquitoes. I. The Anophelines of the Nyssorhynchus group. The American Journal of Hygiene 6: 684-717.

Root, F. M. 1927. Studies on Brazilian mosquitoes III. The Genus Culex. The American Journal of Hygiene 7: 574-598.

Root, F. M. 1927. Studies on Brazilian Mosquitoes. IV. Notes on some 
Brazilian species on Anopheles. The American Journal of Hygiene 7: 599-605.

Rozeboom, L. E. \& W. H. W. Komp. 1950. A review of the species of Culex of the subgenus Melanoconion (Diptera, Culicidae). Annals of the Entomological Society of America 43: 75-114.

Say, T. 1824. Appendix. Part I - Natural history. 1 - Zoology. E. Class Insecta, pp. 268-378. In: Keating, W. H. Narrative of an expedition to the source of St. Peter's River 2 Philadelphia $459 \mathrm{p}$.

Sirivanakarn, S. \& J. N. Belkin. 1980. The identity of Culex (Melanoconion) taeniopus Dyar and Knab and related species with notes on the synonymy and description of a new species (Diptera, Culicidae). Mosquito Systematics 12: 7-24.

Skuse, F. A. A. 1894 (1895). The banded mosquito of Bengal. Indian Museum Notes 3: 20.

Stone, A. 1944. Notes on some Trichoprosopon (Diptera, Culicidae). Revista de Entomologia 15: 335-341.

Tanaka, K. 2003. Studies on the Pupal Mosquitoes of Japan (9). Genus Lutzia, with Establishment of Two New Subgener, Metalutzia and
Insulalutzia (Diptera, Culicidae). Japan Journal of Systematic Entomological 9: 159-169.

Theobald, F. V. 1901. A Monograph of the Culicidae. British Museum of Natural History, Londres Vol. 2, 391 p.

Theobald, F. V. 1903. Two new Jamaican Culicidae. Entomologist 36: 281-283.

Theobald, F. V. 1907. A monograph of the Culicidae or mosquitoes Vol. 4, 639 p.

Theobald, F. V. 1912. No. V. - Culicidae. Transactions of the Linnean Society of London 15: 81-94.

Von Humboldt, F. H. A. 1819. Voyage aux regions equinoxiales du Nouveau Continent, fait en 1799, 1800, 1801, 1802, 1803 et 1804. par Al. De Humboldt et A. Bompland. Vol: 2, 722 pp. In: [Humboldt, F. H. A.., Ed.] Voyage de MM Alexandre de Humoldt et Aime Bompland. Pl. I: Relation historique. Paris.

WRBU. 2007. Systematic Catalog of Culicidae. Washington DC. Walter Reed Biosystematics Unit, Smithsonian Institution., disponível em http://www.mosquitocatalog.org/main.asp, acessado em Junho de 2007.

Recebido em 14/09/2007; aceito em 25/02/2008 